\title{
An epidemiological model for the dynamics of Chagas' disease
}

\author{
Jorge X. Velasco-Hernández \\ Cornell University 322 Warren Hall, Ithaca NY 14853 \\ Universidad Autonoma Metropolitana Xochimilco, Departamento El Hombre y su Am- \\ biente, México.
}

\begin{abstract}
A$ model for the transmission dynamics of Chagas' disease is presented. The structure of the model is similar to that of the RossMacdonald model for malaria but includes an extra infectious compartment (chronically ill individuals) which is characteristic of Chagas' disease. In Chagas' disease there are two main forms of transmission which are transmission by blood transfusion and by vector biting. The former is more common in urban environments and the latter is characteristic of rural settings. The characteristic long chronic (frequently asymptomatic) stage of Chagas' disease is potentially a risk factor that could enhance disease transmission by blood transfusion. The model evaluates the relative importance of both transmission modes in populations of constant size.
\end{abstract}

\section{Introduction}

Chagas' disease is a major health problem in several countries of Latin America. The World Health Organization reports this tropical infection (Vector Biology and Control Division, 1989; Training and Information Guide, 1987) as the second major endemic disease after malaria. The population at risk reached around 64 million in 1984 (Zeledón and Rabinovich, 1981) with around 24 million of infected individuals . Chagas' disease or American trypanosomiasis was discovered by Carlos Chagas, a brazilian physician, around 1909 (Chagas, 1911). Chagas linked the disease with the insect that transmits it and identified other alternative routes of transmission as, for example, congenital transmission. Chagas' disease is characterized by the existence of one acute short duration stage and a chronic stage that can last from 10 to 20 years (Texeira, 1979; Coura, 1988; García and Bruckner, 1988) After this long period the disease can develop into three forms. One is the asymptomatic form in which individuals are seropositive for $T$. cruzi antibodies but present no noticeable symptoms and appear to be in good health; the second form is characterized by the presence of megasyndromes 
or pathological hypertrophy of portions of the digestive tract such as colon and esophagus. Finally, the third form develops into miocarditis (Coura, 1988) and is the one with the worst prognosis: individuals affected by this form have a mean life expectancy of two years once diagnosed (Texeira, 1979). The existence of the long chronic stage enhances the likelihood of transmission by blood transfusion especially through tha asymptomatic patients. Also, if the infectivity period of a chronically infected human hosts is comparable to the average duration of the stage, then very high values for the basic reproductive number of the disease may be achieved; that is, the number of expected secundary infections that arise from the introduction of an infective individual in a completely susceptible population is larger that one.

Previous published modeling efforts for the understanding of Chagas' disease dynamics exists but are scarce. Rabinovich and Rossell (1975) developed a computer model to study strategies for vector control but were not concerned, as we are here, with the long term behavior of the disease on the human population nor with the relative weight of the two main forms of disease spread and dynamics: vectorial and blood transfusion transmission. Two more recent papers by Busenberg and Vargas (1989) and Velasco-Hernández (1991a) are the immediate predecessors of this work. Both of these models analyze disease dynamics in human populations whose net growth rate is significantly different from zero and, furthermore, evaluate the relative importance of vectorial and horizontal transmission in the existence and stability of endemic states. Both, however, aggregate in only one compartment the acute and chronic stage.

In this paper we analyze models in which the total host population remains constant in time. From the perspective of the real phenomena this is only a very rough approximation of what actually happens in the field since Chagas' disease develops during long periods of time, 20 or more years, in which the total population may indeed increase. One very important aspect of the disease that has been left out is the existence of very important alternative hosts or reservoirs of $T$. cruzi. Chagas' disease thrives in rural areas of tropical America where human settlements are in close contact with the natural environment. Several wild animal species exist that can carry the parasite without the need of intermediate human hosts as well as domestic or semidomestic animals that serve as links between the so-called wild and human cycles of Chagas' disease transmission (Coura, 1988).

Nevertheless, the models that assume constant population and do not involve other alternative hosts, can be helpful in the description of general trends of the disease, especially the relative importance of vector transmission as opposed to horizontal transmission by blood transfusion, and the effect on the two different infective stages on the existence and stability of endemic and disease-free stages. Let $x$ denote the susceptible proportion of the total population, let $y$ denote the proportion of individuals who are infected and in the acute phase, and let $z$ to represent the proportion of those individuals who are infected but in the chronic phase of the disease. Also consider the vector population divided into susceptible and infected proportions, this last denoted by $v$. 
We assume that both populations remain constant and so $1-v$ is the proportion of susceptible vectors, and $1-y-z=x$, the proportion of susceptible hosts. Let $a$ represent the number of bites per vector per host per unit time; $b$ is the proportion of infected bites that give rise to infections; $c$ is the proportion of bites on infective host that give rise to infections in the vector; $m$ is the ratio of vector numbers to host numbers; $\delta$ is the vector per capita mortality rate; $\mu$ is the proportion of new acute infections that develop into chronic cases; $h$ is the horizontal transmission rate; $\theta$ is the removal rate of chronic individuals. The model is closely related to the Ross-MacDonald model for malaria (Aron and May, 1982) and assumes that encounters between infective vectors and susceptible host occur at random.

Define $\alpha:=a b m$, and $\beta:=a c$. For easier reference we write the model with the parameters just defined. The equations of the model are as follows

$$
\begin{aligned}
& y(t)=\alpha(1-y(t)-z(t)) v(t)-\mu y(t)+h z(t)(1-y(t)-z(t)), \\
& z^{\prime}(t)=\mu y(t)-\theta z(t), \\
& \mho(t)=\beta(y(t)+z(t))(1-v(t))-\delta v(t)
\end{aligned}
$$

with initial conditions $y(0)=y_{0}, z(0)=z_{0}, v(0)=v_{0}$, and the two auxiliary conditions $x(t)=1-y(t)-z(t)$ and $w(t)=1-v(t)$, where $w(t)$ denotes the susceptible vector population.

\section{Analysis of the vector-host model}

Notice that in this model we are assuming that the fraction of infected vectors is in direct proportion of the fraction of total infected hosts. This means that the fraction of infected vectors is small when compared with the total vector population. If the proportion $v$ were near one, then a more appropriate model would be to consider $v$ as constant, as in the model analyzed by Busenberg and Vargas (1989). It is a simple task to verify that the model is well-posed and that all solutions are non-negative and, thus, biologically feasible.

Setting the right hand sides of (1) equal to zero we derive the following set of equations that determine the values of the coordinates of the equilibria which we denote by $\left(v^{*}, z^{*}\right)$.

$$
\begin{aligned}
& v=\frac{\beta \lambda z}{\delta+\beta \lambda z} \\
& v=\frac{z(\theta-h+h \lambda z)}{\alpha(1-\lambda z)}
\end{aligned}
$$

The second equation in (2) is monotonically increasing in the region where $v>0$ and has an asymptote at $z=\lambda^{-1}<1$. Furthermore, when $\theta-h \leq 0$ a unique positive solution to (2) exists and, in the case where $h<\theta$, solution exists only if the basic reproductive number $R_{0}=\frac{\alpha \beta \lambda}{\delta(\theta-h)}>1$ which gives average the 
number of infections that an infective individual produces during the length of the infectious period, when introduced in an all susceptible population. Thus we have the following

Result 1 If $\theta \leq h$, then there always exist a unique endemic equilibrium point $\left(v^{*}, y^{*}, z^{*}\right)$ for system (1). If $\theta>h$ the unique endemic equilibrium point exist only if the quantity $R_{0}=\frac{\alpha \beta \lambda}{\delta(\theta-h)}$ is larger than one.

In order to study the stability of the endemic (non-trivial) equilibrium point we use the equivalent system of equations which is obtained by using $x=1-y-z$,

$$
\begin{aligned}
& x^{\prime}=-[\alpha x v+h z x-\theta z], \\
& z^{\prime}=\mu(1-x-z)-\theta z, \\
& v=\beta(1-x)(1-v)-\delta v
\end{aligned}
$$

The Jacobian matrix evaluated at the equilibrium point is

$$
A=\left(\begin{array}{ccc}
-\alpha v^{*}-h z^{*} & -h x^{*}+\theta & -\alpha x^{*} \\
-\mu & -(\theta+\mu) & 0 \\
-\left(1-v^{*}\right) \beta & 0 & -\beta\left(1-x^{*}\right)-\delta
\end{array}\right)
$$

To determine sufficient conditions for the local asymptotic stability of the endemic equilibrium we look at two cases. The first requires $h \geq \theta$, and assumes that the condition

$$
x^{*} \geq \frac{\theta}{h}
$$

and the condition

$$
x^{*}<v^{*} \quad x^{*}<z^{*}
$$

hold. Note that $h^{-1}$ can be interpreted as the average length of the infectious period of a blood donor. Thus, condition (3) says that in equilibrium, the susceptible fraction of the population must be greater than the proportion of chronically infected individuals removed from the population during the average duration of infectiuosness of a blood donor. If (3) and (4) are satisfied, then $-A$ is a nonnegative matrix. Moreover, its eigenvalues have non-negative real part as can be easily verified from the corresponding characteristic polynomial. holds. Thus, conditions (3) and (4) together imply local asymptotic stability of the endemic equilibrium point (which is the only equilibrium of the system by Theorem 1). Notice also that, in the case

$$
x^{*}=\frac{\theta}{h},
$$

one can guarantee the existence of an eigenvalue $\lambda^{*}=\theta+\mu$ of $-A$, that satisfies $\lambda^{*} \geq\left|\lambda_{k}\right|$, where $\lambda_{k}$ is the kth eigenvalue of the matrix.

We now consider the case $\theta>h$. From Theorem 1 we know that, in this case, there exist two equilibria for the model, namely the disease-free and endemic states. When $\theta>h$, the structure of $A$ is lost and the stability analysis is not so simple. In particular in the stability analysis of the endemic equilibrium point 
$\left(x^{*}, z^{*}, v^{*}\right)$ the multitude of combinations in which the parameters in the model appear prevent a meaningful biological interpretation if one tries to analyze the problem by directly using the expression for $R_{0}$, for example. This fact, made it necessary to provide an extra condition to be satisfied by $A$ in order to give a clear, although not so general, result on the stability of the equilibria.

This extra condition can be justified biological without difficulty and it is not really a restriction within the context of disease dynamics. We require $\mu>\theta$. Recall that $\mu^{-1}$ represents the average length of the acute stage which is very small compared with the length of the chronic stage $\theta^{-1}$. Thus the inequality holds. We have the following

Prediction 1 Suppose that condition (4) holds. Then, if $\theta>h$ and $\mu>\theta$, the endemic equilibrium point of (1) is locally asymptotically stable. If $\theta \leq$ $h$, the unique equlibrium point $\left(x^{*}, z^{*}, v^{*}\right)$ is locally asymptotically stable.

As a summary of the previous results we present the following result whose proof can be found in Velasco-Hernández (1991b).

Result 2 For system (1) the behavior of the equilibria is as follows:

1. When $\theta \leq h$ the origin is unstable and the non-trivial positive equilibrium point is locally asymptotically stable if condition (4) holds.

2. When $\theta>h$ we have two cases.

2.1 If $R_{0}>1$ the unique non trivial equilibrium point is locally asymptotically stable, if condition (4) holds, and the origin is locally unstable.

2.2 If $R_{0} \leq 1$ the origin is the unique equilibrium point of the system and it is locally asymptotically stable.

\section{Introducing removal rates in both infective stages.}

A variant of the previous model can be constructed to evaluate and compare the qualitative behavior of the spread of the disease when both infective stages are aggregated and collapsed into only one. Assume that the horizontal transmission of the disease is not only dependent of the contacts between chronically infected and susceptible individuals, but also depends on the contacts between individuals in the acute stage and susceptibles. Assume also that there is removal of individuals from the acute stage of the disease. This removal rate can actually be thought as a rough approximation to the phenomenon of superinfectivity (Aron and May, 1982). It seems that infected individuals in endemic regions are subject to continuous attacks by vectors thus potentially producing a new infection when one is already present. This process could be the cause of the onset of the acute stage of the disease in endemic areas (Coura, 1988). Here we assume that the rate of reversion to the uninfected state (Aron and May, 1982) is equal to the rate of removal of chronically infected individuals, given by $\theta$. Keeping the meaning of the parameters used in the previous model we have 


$$
\begin{aligned}
y(t) & =\alpha v(t)(1-y(t)-z(t))-\mu y(t) \\
& +h(y(t)+z(t))(1-y(t)-z(t))-\theta y(t) \\
z^{\prime}(t) & =\mu y(t)-\theta z(t) \\
v^{\prime}(t) & =\beta(y(t)+z(t))(1-v(t))-\delta v(t) .
\end{aligned}
$$

By adding up the first and second equations, we reduce the model to a two dimensional system by defining $i(t)=y(t)+z(t)$, the total proportion of infected individuals regardless of the stage of the disease. Thus we have

$$
\begin{aligned}
& i^{\prime}(t)=(1-i(t))[\alpha v(t)-h i(t)]-\theta i(t) \\
& v(t)=\beta i(t)(1-v(t))-\delta v(t) .
\end{aligned}
$$

We proceed now to the analysis of the asymptotic behavior of (5). First we compute the feasible equilibrium points $\left(i^{*}, v^{*}\right)$ which satisfy:

$$
\begin{aligned}
& v=i \frac{\theta-h(1-i)}{\alpha(1-i)} \\
& v=\frac{\beta i}{\delta+\beta i} .
\end{aligned}
$$

It can be proved (Velasco-Hernández, 1991b) that for model (5) an endemic equilibrium always exists and it is unique provided $\theta \leq h$. If, on the contrary, $\theta>h$ we have two cases: If $R_{0}=\frac{\alpha \beta}{\delta(\theta-h)}$ is greater that one, there exist a unique endemic equilibrium point. If, on the contrary, $R_{0} \leq 1$ then the only equilibrium point is the disease-free state.

The quantity $R_{0}$ is the basic reproductive number for these model equations. Notice that the only difference batween this reproductive number and the one for model (1) is that there is no dependence on the parameter $\lambda$ that measures the net recruitment rate of individuals into the chronic stage of the disease. In this new variant of the model the spread of the disease depends essentially on the vector population.

The stability analysis of the model is straightforward and guaranties the global asymptotic stability of the endemic equilibrium when it exists. The reader is referred to Velasco-Hernández (1991b) for the technical details of the proof.

Prediction 2 The asymptotic behavior of (5) is as follows.

1. If $\theta<h$ then the origin is unstable and the unique non-trivial equilibrium is globally asymptotically stable.

2. If $\theta \geq h$ then there are two cases.

2.1 If $R_{0}>1$, then the origin is unstable and the unique endemic equilibrium point is globally asymptotically stable.

2.2 If $R_{0} \leq 1$ then the disease-free equilibrium is globally asymptotically stable. 


\section{Endemism in the absence of horizontal transmission}

In rural communities where Chagas' disease is endemic, the most important mode of transmission is through vector biting (Zeledón and Rabinovich, 1981; Coura, 1988). Transmission through blood transfusions can be considered absent although its importance must be considered in urban or semi-urban locations (Pinto Dias and Brener, 1984; Pinto Dias et al., 1985; Platero et al. 1983). In a later section we will analyze the case when horizontal transmission can not be neglected and, for the moment, we take the rate of horizontal transmission of the disease to be zero.

For the case $h=0$ in model (1) the basic reproductive number is

$$
R_{0}:=\frac{\alpha \beta \lambda}{\theta \delta}
$$

with $\lambda:=1+\frac{\theta}{\mu}$.

In terms of the reproductive number, the endemic equilibrium point has components

$$
y^{*}=\theta z^{*} / \mu, \quad z^{*}=\frac{\theta}{\mu \lambda}\left(\frac{R_{0}-1}{R_{0}+\beta / \lambda}\right), \quad v^{*}=\frac{R_{0}-1}{R_{0}+\alpha \lambda / \theta}
$$

where $\beta=a c$ and $\alpha=a b m$ are the effective biting rates of triatominae, that is, those bites that effectively transmit the disease to the vector and to the host respectively.

Notice also that model (1) assumes that deaths occur only at the end of the chronic infective period and that eventually all individuals with the acute Chagas' infection will enter the chronic phase.

From the expressions for the endemic equilibrium we can give the following interpretation of the parameter relations involved.

1. $\frac{\alpha}{\theta}$ is the effective number of bites per vector which transmit the acute Chagas' infection to humans during the length of the chronic phase of the disease.

2. $\frac{\beta}{\delta}$ is the effective number of bites per vector which transmit the infection to susceptible vectors during their life span.

3. $\frac{\theta}{\mu}$ is the fraction of humans already in the chronic stage of the disease that die during the length of the acute infection.

4. $\lambda^{-1}=\frac{\mu}{\theta+\mu}$ is the proportion of hosts in the acute stage on the disease that transfer to the chronic phase during the total duration of Chagas' disease.

We rewrite now $R_{0}$ as

$$
R_{0}=\left(\frac{\alpha}{\theta}\right)\left(\frac{\mu+\theta}{\mu}\right)\left(\frac{\beta}{\delta}\right)
$$


Recall that if $R_{0}>1$ there exist a unique endemic equilibrium point that is asymptotically stable. The condition on $R_{0}$ is equivalent to require that the slope of the isocline $d y / d t=0$ evaluated at the origin be less that the slope of the isocline $d v / d t=0$ also evaluated at the origin. Thus, large values of $\frac{\beta \lambda}{\delta}$ would guarantee the existence of endemic equilibria for Chagas' disease. It is worth noting that the ratio $\frac{\beta \lambda}{\delta}$ is related to the so-called 'MacDonald stability index' (Aron and May, 1982) developed for malaria. In Chagas' disease the 'stability index' depends not only on the biting rate but on the infectivity of those bites to the host population.

It is very likely, as some authors (Coura, 1988, Schoefield, 1980, 1982) point out, that the infectivity of triatominae bites is actually enhanced by the frequency with which a susceptible individual is bitten during his/her life, and that the biting frequency depends on both the infectivity of individual bites and on the number of triatominae allocated in average to the human host, i.e., it depends on the ratio $m$. This effect is not included in the present model.

\subsection{Some quantitative results on the threshold parameters}

Visits by this author to endemic zones in the State of Oaxaca, México, indicate a rough conservative estimate of 5 vector bugs per individual although in regions of Brazil, Argentina and Chile the number of bugs per individual fluctuates between 20 and 45 (Rabinovich and Leal, 1979). Estimates of the biting rate are reported by Rabinovich and Leal (1979). Bites do not necessarily indicate infection. Recall that the insect must defecate near the feeding spot in order to have some positive probability of infection. Thus we count only actual blood meals as our 'effective bites'. Since vectors of Chagas' disease go usualy through five nimphal stages (Rabinovich and Leal, 1979) before reaching reproductive age, and a blood meal is necessary for individuals to moult, we take $a=6$. Since there can be roughly two generations of insects in one year then $\frac{a^{2}}{\delta}=18$, approximately. Now, assume that the mean length of the infectious period $(1 / \theta)$ is 10 years. This renders

$$
R_{0}=180 m \cdot b \cdot c .
$$

If $b=c=0.1$, then $R_{0}=1.8$ which indicates existence of a stable endemic state. However, as it can be easily seen, $R_{0}$ is very sensitive to changes in $b c$. For example, if $b c=0.1$, then $R_{0}=18 m$ which is a very high value for the basic reproductive number. Notice that the equivalent 'stability index' of MacDonald in malaria, $\frac{\beta}{\delta}$, is high but does not determine the type of endemic level that the disease reaches in the host population. In fact, the major contribution for the very high value of $R_{0}$ in Chagas' disease comes from the length of the chronic stage and from the ratio $m$. Notice that if $\theta^{-1}=2$ in the above example, then the value of $R_{0}=1.8 \mathrm{~m}$ would be reduced provided $b c=.1$. Thus, early detection of chronic cases together with vector control measures aimed to reduce the biting 
rate $a$ and the ratio $m$ would eventually lead perhaps not to the eradication of the disease but to its confinement within socially acceptable low prevalence values.

\section{Endemism in the presence of horizontal transmission}

In urban centers Chagas' disease is transmitted by the vector and by contaminated blood through blood transfusions (Coura, 1988; Pinto Dias and Brener, 1984). Migratory movement of the rural poor from highly endemic areas to urban centers has increased during this decade in most countries of Latin America bringing with it, in regions close to Chagas' disease endemic zones, an increase in the incidence and prevalence of the infection in urban populations. (Coimbra, 1988; Coura, 1988; Pinto Dias et al., 1985). Lack of jobs, failed or inexistent agrarian reform, and other complex socio-economic causes are factors motivating this migratory movements. Since we are concerned here only with the mechanisms of transmission of Chagas' disease, we focus on the impact that these migrants have on the troubled health care systems in Latin America and, in particular, to the increasing risk of infection that results from $T$ : cruzi contaminated blood banks. In fact, lack of adequate screening of blood samples have increased the chances of Chagas' disease transmission by blood transfusion. In this section we analyze some quantitative consequences of horizontal transmission on the endemic levels predicted by model (1).

Recall that we have two cases predicted by model (1) as outcome of the interaction vector-host-blood transfusion. First, if the rate of blood transfusion $h$ is greater that or equal to the removal rate of chronically ill individuals $\theta$, then the endemic equilibrium always exist an it is unique and globally asymptotically stable: there is no hope of eradication. The stated condition means that the fraction of susceptible host becoming infected during the average duration of the chronic phase must exceed or be equal to one. When this fraction goes below one we are in the second situation predicted by the model. In this case there exist the possibility of eradication although the value of $R_{0}$ is even higher than when $h=0$, all other parameters held equal. The 'stability index' $\frac{\beta \lambda}{\delta}$ remains the same but the other factor involved changes to $\frac{\alpha}{\theta-h}$.

Thus, in the presence of blood transfusion it is not only important to have early detection of chronic cases and vector control programs but also to diminish $h$. In fact, even if all acute cases are detected promptly, which makes the duration of the chronic phase practically zero, the endemic level of the disease will persist unless $h$ drops nearly to zero. Thus, in urban regions, Chagas' disease transmission must be independent of vector transmission in order to persist in the population.

Model (1) assumes that the removal rate of the acute stage is zero. However this is not a realistic assumption (Coura, 1988; Texeira, 1979). In fact, the onset 
of the acute infection is produced after continuous reinfections of the parasite (Coura, 1988). This phenomenon was modeled, as the reader may recall, by the introduction of a removal rate in the acute stage (superinfectivity) in the system of equations (5). Model (5) also introduces the effect of horizontal transmission but now the recruitment rate of new infecteds by this transmission mode depends in both infective stages.

There are no major changes on the behavior of the net reproductive number $R_{0}$ as compared with the case studied in the previous section. The only change is the removal of the factor $\lambda$ from $R_{0}$ which is a minor modification since its value is always very close to 1 within the range of parameter values feasible for Chagas' disease. Thus, aggregating in one the acute and chronic stages (by means of the introduction of the removal rate in the acute stage) does not affect the net qualitative long term behavior of the disease.

\section{Acknowledgements}

This research was supported by an Academic Scholarship from the Universidad Autónoma Metropolitana Unidad Xochimilco, and partially supported by CONACYT, México. This research has also been partially supported with funds from the Dean of the Office of Sponsored Programs of the College of Agriculture, and the Mathematical Science Institute at Cornell University, and by NSF grant DMS-8906580 to Carlos Castillo-Chávez. The ideas presented in this work arose as a product of the discussions I held, during the last three years, with Stavros Busenberg whom I sincerely thank. 


\section{References}

1 Aron, J. and R. May. 1982, The population dynamics of malaria in Anderson, R. M. (editor) The population Dynamics of Infectious Diseases: Theory and Applications, pp. 139-178, Chapman and Hall, London.

2 Busenberg, S. and C. Vargas. 1988, Modeling Chagas' disease: variable population size and demographic implications. Preprint Harvey Mudd College, California.

3 Chagas, Carlos. 1911, Nova tripanozomiaze humana: Estudos sobre a morfolojia e ciclo evolutivo do Schizotrypanum cruzi n. ge.,n. sp. ajente etiolojico de nova entidade morbida do homem Mem. Inst. Oswaldo Cruz. 1.

4 Coimbra, C.E.A. 1988, Human settlements, demographic pattern, and epidemiology in lowland Amazonia: The case of Chagas' disease. American Antrop. 90: 82-97.

5 Coura, J.R. 1988, Determinantes epidemiológicos da doenca de Chagas no Brasil: A infeccao, a doenca e sua morbi mortalidade. Mem. Inst. Oswaldo Cruz. Supplement 83: 394-402.

6 Garcia, L. and D.A.Bruckner 1988, Diagnostic Medical Parasitology. Elsevier, New York.

7 Gorla, D. E. and C. J. Schofield. 1985, Analysis of egg mortality in experimental populations of Triatoma infestans under natural climatic conditions in Argentina. Bull. Soc. Vector Ecol. 10: 107-117.

8 Pinto Dias, J. C. and S. Brener. 1984, Chagas' disease and blood transfussion. Mem. Inst. Oswaldo Cruz. 79: 139-147.

9 Pinto Dias, J., C. Prates, and S. Brenner. 1985, Doenca de Chagas en Minas Gerais: situacao atual e perpectivas. Ren. Brasil. Malariol. D. Trop. 37: 7-28.

10 Platero, F., C. Flores, M. Contreras, L. Sandoval, P. Salinas, N. Estay, E. Sanchez, 1. Grawe y H. Schenone. 1983, Enfermedad de Chagas en Chile. Sectores Urbanos. V Prevalencia de la infección por Trypanosoma cruzi en donadores de sangre y madres y recién nacidos en el Hospital de Iquique. Bol. Chile. Parasit. 38: 76-78.

11 Rabinovich, J. and J. Leal. 1979, Domiciliary biting frequency and blood ingestion of the Chagas' disease vector Rhodnius prolixus Stahl (Hemiptera: Reduviidae), in Venezuela. Trans. R. Soc. Trop. Med. Hyg. 73: 272-283.

12 Rabinovich, J. E. and O. Rossell. 1975, Mathematical models and ecology of Chagas' disease. In PAHO American trypanosomiasis Research. Sci. Pub 318, 359-369 Washington.

13 Schofield, C. J. 1980, Density regulation of domestic populations of Triatoma infestans in Brazil. Trans. R. Soc. Trop. Med. Hyg.74: 761-769.

14 Schofield, C. J. 1982, The role of blood intake in density regulation of populations of Triatoma infestans (Klug)(Hemiptera: Reduviidae). Bull. Ent. Res. 72: 617-629.

15 Texeira, A. 1979, Chagas' disease: trends in immunological research and prospects for immunoprophylaxis.Bull. WHO 57: 697-710. 
16 Training and Information Guide. 1987, Vector Control Series World Health Organization, Vector Biology and Control Division, Geneva.

17 Vector Biology and Control Division. 1989, Geographical Distribution of Arthropod-borne diseases and their principal vectors. World Health Organization, Geneva.

18 Velasco-Hernández J.X.1991a, Disease Dynamics in Variable populations: The Chagas' Disease case. Preprint. Biometrics Unit, Cornell University.

19 Velasco-Hernández, J.X. 1991b, Analysis of a model for Chagas' Disease with constant Host Population size. Preprint. Biometrics Unit, Cornell University.

20 Zeledón, R. and J. E. Rabinovich 1981, Chagas' disease: An ecological appraisal with special emphasis on its insect vector. Ann. Rev. Ecol. Syst. 26: 101-133. 